\title{
Cannabis use by people with severe mental illness - is it important?
}

\section{Zerrin Atakan}

\begin{abstract}
Cannabis use is more common among people with severe mental illness than in the general population. It has detrimental effects on the course of the illness, physical health and social life of users, as well as being a financial burden on health services. It is important to understand why some people with severe mental illness continue to use cannabis, despite experiencing its effects on their condition. This article reviews research on the scale of cannabis use by such patients, the effects on the course of their illness, possible reasons to explain why they use it, and how they can be assessed in clinical settings, as well as providing some assessment tools to measure various characteristics related to cannabis use.
\end{abstract}

Zerrin Atakan will discuss further management and treatment models for cannabis use by people with severe mental illness in the January 2009 issue of Advances in Psychiatric Treatment.

Cannabis is the most widely used illicit substance in the world (Fig. 1) and its use is more common among people with severe mental illness than in the general population (Green et al, 2005; Boydell et al, 2006). In the UK and some other Western countries, along with the relaxation of legislation and the prevalence of the view that cannabis is a 'harmless' drug, its use has been steeply increasing

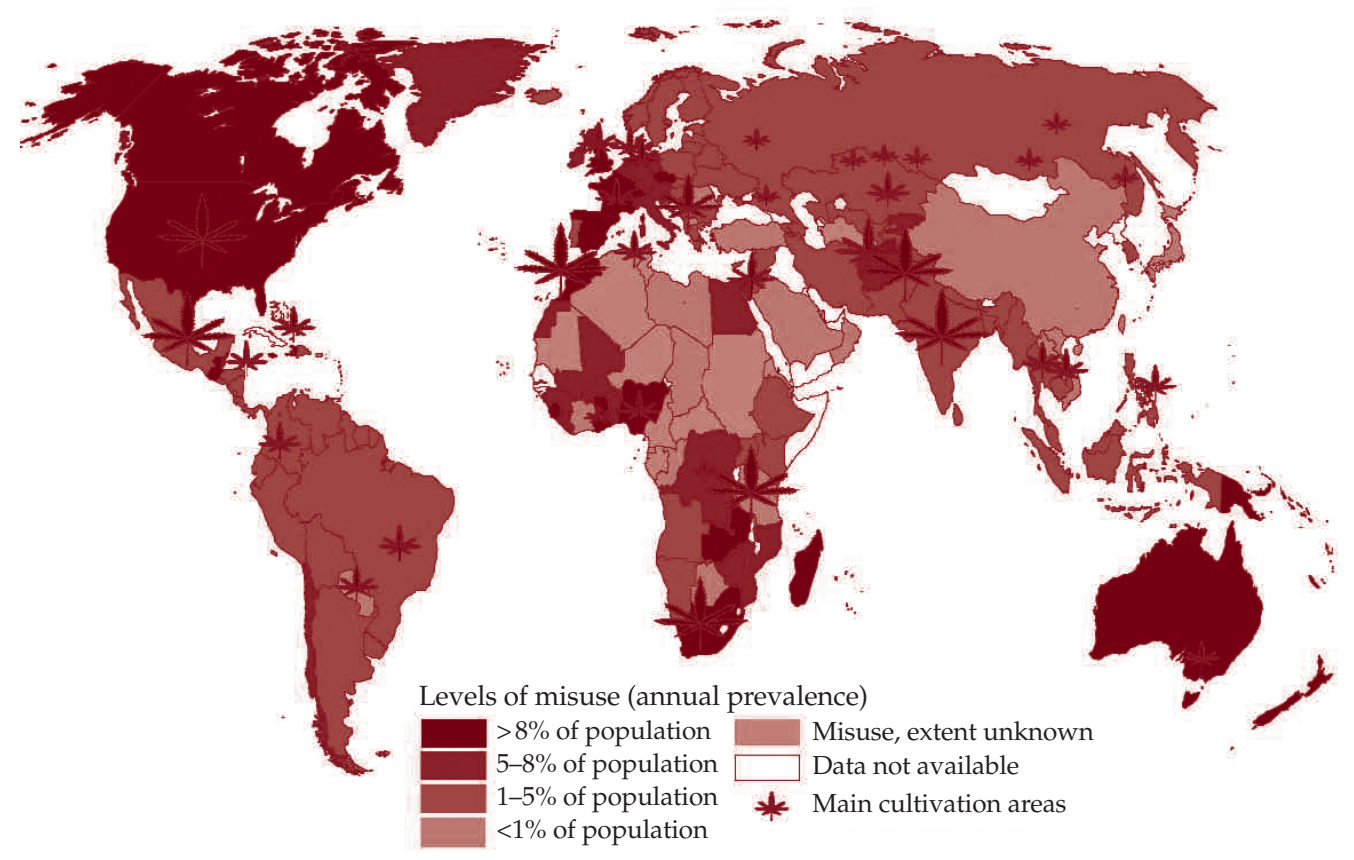

Fig. 1 Use of cannabis in 2003-2004 (or latest year available). After United Nations Office on Drugs and Crime (2006), with permission. A copy of the original map, with clearer definition of areas, appears as Fig. DS1 in an online supplement to this article.

Zerrin Atakan worked as the lead consultant in the National Psychosis Unit at the Maudsley \& Bethlem Royal Hospitals in London until August 2007, when she retired from NHS duties. She currently holds an honorary contract at the Institute of Psychiatry (De Crespigny Park, London SE5 8AF, UK; email: Zerrin.Atakan@iop.kcl.ac.uk), where she is doing research on the effects of cannabis compounds on brain functioning and development of an intervention model for cannabis-using patients with psychosis. As a consultant psychiatrist she gained extensive expertise treating severe mental illness. She has advised the Home Office, voluntary mental health organisations and local councils on cannabis use, and written for cannabis websites to inform young people about the effects of the drug on mental health. 
and the age at first use has been steadily decreasing (Johns, 2001; Degenhardt et al, 2003; Hall \& Degenhardt, 2007). Especially among young people, cannabis use is becoming more common than cigarette smoking, partly owing to the growing social disapproval of tobacco use (Rey \& Tennant, 2002).

Furthermore, the potency of street cannabis has increased over recent decades: it is thought to contain about three times more tetrahydrocannabinol (THC, the major active psychoactive ingredient of the plant) than it did in the 1960s. According to a recent study, even the THC content of 'skunk' has doubled over the past 10 years (Potter et al, 2008). The THC content of cannabis varies according to which part of the plant is used - flowers and top parts of the plant contain more THC than other sections (ElSohly et al, 2000).

In the UK, it is estimated that between 20 and $70 \%$ of people with severe mental illness use cannabis (Miles et al, 2003; Isaac et al, 2005). A review of 53 treatment samples and 5 epidemiological studies examining the prevalence of cannabis use and misuse among people with psychosis reported lifetime use in $42 \%$ and lifetime misuse in $22.5 \%$ (Green et al, 2005). Prevalence of current use was reported to be $23 \%$ and current misuse $11.3 \%$. A UK study involving 115 patients in a psychiatric intensive care unit found that $71.3 \%$ were misusing cannabis (Isaac et al, 2005). These individuals were found to be more severely ill and spent longer periods in hospital care than those who did not use the drug.

Polydrug misuse is also common and underdetection of substance use can be as high as 50\% (Kavanagh et al, 2002). Furthermore, people with severe mental illness are more likely to be dependent on cannabis (Degenhardt \& Hall, 2001). Consequently, there has been a justified interest in comorbidity (dual diagnosis), owing to its high prevalence and significant impact on clinical and social problems, with a resultant heavy financial burden on health services. This interest has expanded into research specifically studying the association between the use of cannabis by young people and the subsequent risk of developing a severe mental illness (Arseneault et al, 2002; van Os et al, 2002; Zammit et al, 2002; Fergusson et al, 2003; Henquet et al, 2005).

A recent systematic review of longitudinal and population-based studies showed that cannabis use significantly increases the risk of developing a psychotic illness and that the increase is dosedependent (Moore et al, 2007). The same review did not find strong evidence that cannabis use is more harmful in younger age groups but recommended further studies on this. Macleod, however, writing in Advances in Psychiatric Treatment, critically examined the scientific evidence of a causal association between cannabis use and psychosis and concluded that, at present, evidence for this association is not strong. Nevertheless, he calls for better observational and genetic studies and for interventions targeting cannabis use, especially in the young (Macleod, 2007).

Because cannabis use is highly prevalent among young people, a considerable number of studies have examined the link with first-episode psychosis. In one such study, it was found that substance use among a sample of 123 patients was twice that in the general population, and was more common in men than in women (Barnett et al, 2007). Cannabis misuse was reported in $51 \%$ of the sample and alcohol misuse was seen in 43\%. Age at first use of cannabis was significantly associated with age at first psychotic symptom. Another study, involving 152 individuals with first-episode schizophrenia, reported at baseline that $63 \%$ of participants had at some time used cannabis and that $32 \%$ were currently using the drug (Harrison et al, 2007). At the follow-up assessment this figure had dropped to $18.5 \%$, but those still using the drug presented with significantly more severe positive and depressive symptoms and a greater overall severity of illness. In a 3-year longitudinal study involving 203 admissions to an early psychosis programme, high rates of substance misuse, particularly cannabis, were found and there was again a significant association with male gender, young age and age at onset of psychosis, and positive symptoms (Addington \& Addington, 2007).

Box 1 summarises the key points of this section.

\section{Box 1 The scale of cannabis use}

- Cannabis use is increasing worldwide

- Age at first use is falling

- People with severe mental illness use cannabis more than the general population and are more likely to be dependent on it

- Cannabis use is significantly more common among people with first-episode severe mental illness

- Cannabis use significantly increases the risk of developing a psychotic illness, and the heavier the use, the higher the risk

- There is as yet no strong evidence indicating that using cannabis at a young age is more harmful for the development of a severe mental illness 


\section{The effect of cannabis on severe mental illness: why is it important?}

There is a complex interaction between severe mental illness and the use of substances such as cannabis. There is ample evidence that cannabis use can have major detrimental effects on the course of the illness when patients continue to use the drug (Mueser et al, 2000; Arseneault et al, 2002). As well as worsening the outcome and exacerbating the symptoms, cannabis use by people with severe mental illness can lead to behavioural disturbances such as increased risk of violence and criminal activity (Miles et al, 2003). It is not uncommon in clinical settings to observe a sudden increase in suspiciousness and hallucinatory behaviour when a patient with severe mental illness uses cannabis (Sorbara et al, 2003).

Ongoing substance use has been associated with 'revolving-door' admissions of people with psychiatric disorders, with admissions for users being nearly twice as frequent as those for nonusers (Haywood et al, 1995; Menezes et al, 1996). Furthermore, users' admissions are reported to be longer, with an increase in behavioural disturbances. Medication non-adherence is also strongly associated with substance use among patients with schizophrenia (Owen et al, 1996).

Follow-up studies indicate that persistent substance use after first admission for psychosis has a deleterious impact on clinical outcome. This includes increased risks of readmission, of exacerbation of psychotic symptoms and of a more continuous course of illness (Sorbara et al, 2003). In another outcome study, comparing adolescents and adults with first-episode psychosis, it was shown that the adolescents used more cannabis than the adults and had a greater number of relapses (Pencer et al, 2005). Continuing cannabis use also adversely affects the physical health of people with severe mental illness: for example, they tend to have higher blood glucose levels than those who do not use cannabis (Isaac et al, 2005). The cannabis plant contains several of the same carcinogens as tobacco and it is usually smoked by mixing it with tobacco. Concerns have therefore been raised that cannabis presents an additional risk factor for tobacco-related cancers (Hashibe et al, 2005).

Because persistent use of cannabis is associated with poorer prognosis, it is not surprising that it increases the burden of ill heath not only on patients and their family or carers, but also on health services called on to provide frequent inpatient treatments and more intensive community care (Williams et al, 2005).
Box 2 Associations between severe mental illness and cannabis use

The co-occurrence of severe mental illness and cannabis use has been associated with:

- early psychotic breakdown

- exacerbation or precipitation of symptoms

- poor adherence to treatment

- increased rates of hospitalisation

- increased duration of hospitalisation

- increased duration of psychotic episode

- poor social functioning

- increased rates of violence

- increased rates of suicide

- increased rates of victimisation

- homelessness

- criminal behaviour

- poorer physical health

- heavy burden on health services

It is important to understand that substance use can arise from the adverse social circumstances in which individuals with severe mental illness commonly find themselves (Drake et al, 2008). The negative interactions that arise from this combination can be further worsened by lack of efficient treatment models.

Box 2 summarises the key points of this section.

\section{Why is cannabis use in severe mental illness so common?}

I have already indicated that people with severe mental illness use cannabis more than the general population. It is important to understand why so many specifically choose cannabis and continue to use it even when they experience its negative effects, so that specific 'causes' of cannabis use are appropriately targeted during interventions.

\section{What do patients say?}

In my own clinical experience, most people with severe mental illness who use cannabis give the same reasons as anyone else who uses the drug: for example, cannabis 'relaxes' them, they 'enjoy the buzz' it gives, it makes them feel 'part of a social setting'; in addition, however, they also say that cannabis is 'good for the voices they hear'. Some patients will admit that cannabis can make them feel 'paranoid', especially at the beginning 
of a relapse or admission, when the effect of the drug is still fresh in their mind. However, when they recover from psychotic symptoms, most will 'forget' the negative effects and go back to smoking it when they get a chance.

\section{What do researchers say?}

One possible explanation for cannabis use by patients with severe mental illness is the selfmedication theory. This hypothesises that psychiatric symptoms are alleviated by cannabis or that cannabis is used to counter the side-effects of psychotropic medication. However, the theory has yet to be confirmed by research.

The first study to make a comprehensive examination of subjective experiences of cannabis use and reasons for using the drug among a group of people with psychosis found that the primary reason given was related to neither positive or negative symptoms, nor obvious side-effects of medication (Schofield et al, 2006). Instead, the most frequently cited motivations were to reduce boredom, improve socialisation and alleviate a cluster of symptoms, the most common of which were inner unrest/agitation (47\%) and difficulty sleeping (43\%). Consistent with other research, the participants also reported that cannabis 'took away the feelings of depression'. Only about $10 \%$ reported using it to reduce positive symptoms such as suspiciousness. Schofield and colleagues suggest that the results of other studies examining the link between substance use and severe mental illness indicate three main motives for use: to relax and reduce boredom; to socialise; and to enjoy the intoxication or to enhance positive mood.

Mueser et al (1998) suggest a number of models to explain the relationship between substance use disorder and severe mental illness.

- Secondary substance use disorder model Patients with severe mental illness are particularly vulnerable to developing substance use disorder. The two main hypotheses explaining the transition are psychosocial risk factors and biologically based heightened sensitivity.

- Secondary psychiatric disorder model Severe mental illness is the result of substance use disorder. The proposition that cannabis can 'cause' severe mental illness belongs to this model.

- Common-factor model Both severe mental illness and substance use disorder share the same or similar risk factors (e.g. genetic loading, antisocial personality disorder, socioeconomic status and cognitive functioning).
- Bidirectional model Either disorder can increase the individual's vulnerability to the other, and they are mutually maintaining, with an ongoing interaction between them.

Most of the existing research has been carried out on the first two models; the other two remain untested.

Gregg et al (2007) provide a detailed and comprehensive review of the latest literature on reasons for increased substance use by people with severe mental illness. They found that selfreport studies support an 'alleviation of dysphoria' model of use, but the support for the selfmedication hypothesis is limited. They conclude that common-factor models and bidirectional models of comorbidity do not yet reach validity. They discuss the existence of multiple risk factors in the interaction between substance use and severe mental illness and make further comments on the need to develop and test a multiple risk factor model.

\section{Can cannabis be good for psychotic symptoms?}

Interestingly cannabidiol, one of the compounds in the cannabis plant, has been found to have antipsychotic properties, as well as having antianxiety and anticonvulsive effects, both in animals and humans (Mechoulam et al, 2002; Ashton et al, 2005; Zuardi et al, 2006). In fact, the effects of THC, the main active psychoactive ingredient of the plant, are significantly reduced by cannabidiol (Zuardi et al, 2006). A trial comparing cannabidiol with the antipsychotic amisulpride on people with schizophrenia reported that cannabidiol was as effective as amisulpride in treating psychotic symptoms and that fewer and less severe sideeffects were observed in the cannabidiol group (Leweke et al, 2007).

In view of cannabidiol's potent anxiolytic effect, it is possible that it relieves dysphoria and anxious states in people with severe mental illness. It might also 'treat' some of their psychotic experiences. However, it is unlikely that the cannabis now used in most countries contains sufficient amounts of cannabidiol, owing to the popularity of high-THC varieties of the plant. For instance, the South African cannabis known as dagga has a very high THC content and is almost devoid of cannabidiol. Hydroponically grown 'skunk' also has very small quantities of cannabidiol. This is due to chemical competition between the two compounds within the plant.

For key points see Box 3 . 
Box 3 Why do people with severe mental illness use cannabis?

- The main reasons given for cannabis use are to reduce boredom, to socialise and to enjoy the positive mood that intoxication provides

- Nearly half of patients use cannabis to get relief from dysphoria and agitation and to sleep better; a minority use it to reduce their suspiciousness

- Self-medication has been proposed as a reason for cannabis use, but this is yet to be supported by research evidence

- The cannabidiol in cannabis has strong antianxiety and antipsychotic effects, but street cannabis now contains very small amounts of cannabidiol

- None of the models proposed to explain the interaction between severe mental illness and substance use disorder has a satisfactory evidence base, and it is thought that a multiple risk factor model is needed

\section{How can cannabis use be managed?}

I have already outlined the complexity of the interaction between cannabis use and severe mental illness, as well as the various problems related to ongoing cannabis use by people with severe mental illness. The problem is unfortunately worsened further by the lack of effective, evidence-based treatments. However, over the past 5 years or so this has been recognised as a significant problem and initiatives have been taken at various levels, ranging from governmental to voluntary organisations. In 1999, an article in the BMJ warned that comorbidity is an ever-increasing problem (Weaver et al, 1999). The authors recommended investment in an appropriately skilled workforce, further research initiatives, and development and testing of new models of service delivery for widespread implementation. Since that time, the Department of Health in the UK published two White Papers on dual diagnosis (Department of Health, 2002, 2006). Clinical practice guidelines on the treatment of substance use disorders published by the American Psychiatric Association (Kleber et al, 2007) do not address comorbidity, but they include recommendations for treating cannabis use disorder.

Regardless of the available guidelines, the care and treatment of patients with severe mental illness who continue to use cannabis is still in the early development phase and there is limited research evidence for successful models of management. Few studies of substance misuse have focused on cannabis, and some believe that more substancespecific research is required, as different substances can be associated with different personality traits and genetic influences (Grekin et al, 2006; Young et $a l, 2006)$. Characteristics associated with cannabis use and severe mental illness may require specific management strategies.

Given this situation, the strategies discussed below are based on research examining treatment models for dual diagnosis in general.

\section{Assessment of use}

Comprehensive assessment is the first step towards effective management and treatment of coexisting severe mental illness and substance misuse. It is not enough simply to note whether the patient uses or has used cannabis. A thorough assessment will provide a better understanding of the interactions between the two conditions and why a particular person has chosen to use the drug. Furthermore, a careful assessment of the presence of motivators for cannabis use, as discussed above, is essential, so that precipitating factors are eliminated or reduced.

Assessment scales with proven validity in severe mental illness are available (see next section), but for practical reasons it is not always possible to use them. Box 4 lists the items that a comprehensive history of cannabis use should include. Some

Box 4 Elements of a comprehensive history of cannabis use

- Age at first cannabis use

- Duration of cannabis use

- Amount of use over a set period of time

- Type and strength of cannabis used over the period of time

- Preferred method of use

- Reasons for use, including what the patient gains or loses from it

- Subjective experiences and whether these have changed over time

- Effects on mental and physical health, social life, medication, treatment adherence and finances, as experienced by the patient

- Other substance use (same information gathering for each substance used) 
electronic database systems allow more detailed information gathering for this purpose. While making an assessment, it is important to maintain a non-judgemental manner. Underreporting is very common and further information should be gathered from carers and friends, if possible.

The use of cannabis and other substances can continue in acute care settings, despite efforts to prevent it, and can have detrimental effects on treatment progress. The associated deterioration in clinical state can demoralise not only the patients themselves, but also the staff caring for them. It is important to acknowledge the possibility of continuing use without losing the determination to help the patient to change their drugtaking behaviour. Having an open and honest communication about these matters will encourage the patient to be more open about their reasons for drug use. It is crucial to engage the patient in all steps of care by ensuring their active involvement in their treatment. This includes drawing up care plans with the patient, which may include preventive measures such as agreeing to talk about their craving and to accept randomised urine drug analyses.

\section{Assessment tools}

Scales for assessing and measuring substance misuse specifically by people with severe mental illness have been developed only over the past decade or so. These tools can be used for a more thorough assessment to ensure the collection of good-quality data. The Psychiatric Research Interview for Substance and Mental Disorders (PRISM) has been shown to be reliable, with enhanced diagnostic accuracy (Hasin et al, 1996), but it is time-consuming. The Chemical Use, Abuse and Dependence (CUAD) scale, on the other hand, is shorter and also has proven validity and reliability (Appleby et al, 1996).

A number of assessment tools that are normally used for non-psychotic populations have been tried on people with coexisting severe mental illness and substance use problems. For instance, the Addiction Severity Index, which has wellestablished reliability and validity (McLellan et al, 1980), has been revised to include psychiatric problems (McLellan et al, 1992) and has been found to be a reliable method of assessing drug and alcohol use among patients with psychotic disorders (Helseth et al, 2005).

Some scales provide information on the individual's level of motivation to change. One such is the Stages of Change Readiness and Treatment Eagerness Scale (SOCRATES), which is a 19-item self-report measure (Miller \& Tonigan, 1996). In a study that compared various readiness-to-change scales, Carey et al (2001) found that they had validity and were stable over time. The authors stated that such scales support efforts to quantify readiness to change substance misuse behaviour in people with severe mental illness, but they should not be used exclusively.

The Substance Abuse Treatment Scale (SATS) evaluates treatment progress and outcome. Its validity and reliability have been shown in a community-based sample of people with severe mental illness (McHugo et al, 1995).

Scales such as the Substance Use Scale for Psychosis (Spencer et al, 2002) and the Readiness to Change Questionnaire (James et al, 2004) can be used to explore patients' reasons for using drugs and their level of motivation to change. The Alcohol Timeline Followback, which was originally developed to assess drinking patterns, can be used to obtain retrospective estimates of cannabis use. It has high validity and good measurement properties for estimates of use over periods of up to 12 months before the interview date (Sobell \& Sobell, 1992; Sobell et al, 1996).

The Cannabis Experiences Questionnaire is a recently developed instrument for exploring subjective experiences of cannabis use (Barkus et $a l, 2006)$. The questionnaire has three sub-scales: Pleasurable Experiences, Psychosis-Like Experiences and After-Effects. Barkus et al found that people with high schizotypal personality scores (on the Schizotypal Personality Questionnaire) were more likely to report both psychosis-like experiences and unpleasant after-effects associated with cannabis use.

\section{Psychoeducation on the effects of substances}

The impact of promoting healthy choices and early education on the effects of tobacco smoking has led to a steady decrease in its use in the UK (Henry et al, 2003). However, despite the noticeable increase in cannabis use, especially among the young, there have been no successful campaigns informing people of the health effects of the drug. Education, especially as a preventive measure, is particularly crucial given the available evidence on the harmful effects of cannabis in triggering an enduring psychotic illness, particularly in those who have a predisposition to psychosis.

The evidence base on the effects of psychoeducation on substance use for people with severe mental illness is limited. However, a review of studies of smoking cessation programmes for people with mental illnesses concluded that the majority of successful interventions combined 
psychoeducation and medication (El-Guebaly et al, 2002). Providing information to people with severe mental illness on the effects of substance use and how it interacts with their treatment and illness outcome can be a daunting task, as many patients cannot easily retain this information. Nevertheless, Macpherson et al (1996) found that well-structured educational sessions could influence patients' insight into their mental health problems. The authors suggest that a single session is insufficient and that a series of sessions is needed to consolidate learning. Carers' involvement is crucial and they too require information on all aspects of mental illness and the impact of substance use. Further work needs to be done in this area to provide evidence of the impact of psychoeducation on harm reduction or cessation of substance use.

\section{Conclusions}

Nearly half of people with severe mental illness use cannabis at some time during their lives, and the impact of increasingly potent street cannabis on the course of the illness is complex, multiple and severe. In this article I have attempted to show the importance of identifying cannabis use in these patients and of understanding why some continue to use it, even though they experience the detrimental effects it has on them. In my next article (Atakan, 2009) I will examine psychosocial and pharmacological treatment models for such patients.

\section{Declaration of interest}

None.

\section{References}

Addington, J. \& Addington, D. (2007) Patterns, predictors and impact of substance use in early psychosis: a longitudinal study. Acta Psychiatrica Scandinavica, 115, 304-309.

Appleby, L., Dyson, V., Altman, E., et al (1996) Utility of the Chemical Use, Abuse and Dependence scale in screening patients with severe mental illness. Psychiatric Services, 47 , 647-649.

Arseneault, L., Cannon, M., Murray, R., et al (2002) Cannabis use in adolescence and risk for adult psychosis: longitudinal prospective study. $B M J, 325,1212-1213$.

Ashton, C. H., Moore, P. B., Gallagher, P., et al (2005) Cannabinoids in bipolar affective disorder: a review and discussion of their therapeutic potential. Journal of Psychopharmacology, 19, 293-300.

Atakan, Z. (2009) Managing cannabis use in people with severe mental illness: what can be done? Advances in Psychiatric Treatment, 15, in press.

Barkus, E. J., Stirling, J., Hopkins, R. S., et al (2006) Cannabisinduced psychosis-like experiences are associated with high schizotypy. Psychopathology, 39, 175-178.

Barnett, J. H., Werners, U., Secher, S. M., et al (2007) Substance use in a population-based clinic sample of people with firstepisode psychosis. British Journal of Psychiatry, 190, 515-520.
Boydell, J., van Os, J., Caspi, A., et al (2006) Trends in cannabis use prior to first presentation with schizophrenia in SouthEast London between 1965 and 1999. Psychological Medicine, 36, 1441-1446.

Carey, K. B., Maisto, S. A., Cary, M. P., et al (2001) Measuring readiness-to-change substance misuse among psychiatric outpatients: I. Reliability and validity of self-report measures. Journal of Study on Alcohol, 62, 79-88.

Degenhardt, L. \& Hall, W. (2001) The association between psychosis and problematic drug use among Australian adults. Findings from the National Survey of Mental Health and Well-Being. Psychological Medicine, 31, 659-668.

Degenhardt, L., Hall, W. \& Lynskey M. (2003) Testing hypotheses about the relationship between cannabis use and psychosis. Drug and Alcohol Dependence, 71,37-48.

Department of Health (2002) Dual Diagnosis in Mental Health Inpatient and Day Hospital Settings. Department of Health.

Department of Health (2006) Dual Diagnosis in Mental Health Inpatient and Day Hospital Settings. Department of Health.

Drake, R. E., O'Neal, E..L. \& Wallach, M. A. (2008) A systematic review of psychosocial research on psychosocial interventions for people with co-occurring severe mental and substance use disorders. Journal of Substance Abuse Treatment, 34, 123-138.

El-Guebaly, N., Cathcart, J., Currie, S., et al (2002) Smoking cessation approaches for persons with mental illness or addictive disorders. Psychiatric Services, 53, 1166-1170.

ElSohly, M. A., Ross, S. A., Mehmedic, Z., et al (2000), Potency trends of delta9-THC and other cannabinoids in confiscated marijuana from 1980-1997. Journal of Forensic Sciences, 45, 24-30.

Fergusson, D. M., Horwood, L. J. \& Swain-Campbell, N. R. (2003) Cannabis dependence and psychotic symptoms in young people. Psychological Medicine, 33, 15-21.

Green, B., Young, R. \& Kavanagh, D. (2005) Cannabis use and misuse prevalence among people with psychosis. British Journal of Psychiatry, 187, 306-313.

Gregg, L., Barrowclough, C. \& Haddock, G. (2007) Reasons for increased substance use in psychosis. Clinical Psychology Review, 27, 494-510.

Grekin, E. R., Sher, K. J. \& Wood, P. K. (2006) Personality and substance dependence symptoms: modelling substancespecific traits. Psychology of Addictive Behaviours, 20, 415-424.

Hall, W. \& Degenhardt, L. (2007) Prevalence and correlates of cannabis use in developed and developing countries. Current Opinion in Psychiatry, 20, 393-397.

Harrison, I., Joyce, E. M., Mutsatsa, S. H., et al (2007) Naturalistic follow-up of co-morbid substance use in schizophrenia: the West London first-episode study. Psychological Medicine, 38, 79-88.

Hashibe, M., Straif, K., Tashkin, D. P., et al (2005) Epidemiologic review of marijuana use and cancer risk. Alcohol, 35, 265275.

Hasin, D. S., Trautman, K. D. \& Miele, G. M. (1996) Psychiatric Research Interview for Substance and Mental disorders (PRISM): reliability for substance abusers. American Journal of Psychiatry, 153, 1195-1201.

Haywood, T. W., Kravitz, H. M., Grossman, L. S., et al (1995) Predicting the 'revolving door' phenomenon among patients with schizophrenic, schizoaffective and affective disorders. American Journal of Psychiatry, 152, 856-861.

Helseth, V., Lykke-Enger, T., Aamo, T. O., et al (2005) Drug screening among patients aged 17-40 admitted with psychosis. Tidsskrift for den Norske Lægeforening, 4, 1178-1180.

Henquet, C., Krabbendam, L., Spauwen, J., et al (2005) Prospective cohort study of cannabis use, predisposition for psychosis and psychotic symptoms in young people. BMJ, 330, 11 .

Henry, J. A., Oldfield, W. L. \& Kon, O. M. (2003) Comparing cannabis with tobacco. BMJ, 326, 942-943.

Isaac, M., Isaac, M. \& Holloway F. (2005) Is cannabis an antiantipsychotic? The experience in psychiatric intensive care. Human Psychopharmacology, 20, 207-210.

James, W., Preston, N. J., Koh, G., et al (2004) A group intervention which assists patients with dual diagnosis reduce their drug use: a randomized controlled trial. Psychological Medicine, 34, 983-990. 
Johns, A. (2001) Psychiatric effects of cannabis. British Journal of Psychiatry, 178, 116-122.

Kavanagh, D. J., McGrath, J., Saunders, J. B., et al (2002) Substance misuse in patients with schizophrenia: epidemiology and management. Drugs, 65, 743-755.

Kleber, H. D., Weiss, R. D., Anton, R. F. Jr., et al (2007) Treatment of patients with substance use disorders, second edition. American Psychiatric Association. American Journal of Psychiatry, 164 (suppl. 4), 5-123.

Leweke, F. M., Koethe, D., Gerth, C. W., et al (2007) Cannabidiol as an antipsychotic agent. European Psychiatry, 22 (suppl. 1), S21.

Macleod, J. (2007) Cannabis use and psychosis: the origins and implications of an association. Advances in Psychiatric Treatment, 13, 400-411.

Macpherson, R., Jerrom, B. \& Hughes, A. (1996) A controlled study of education about drug treatment in schizophrenia. British Journal of Psychiatry, 168, 709-717.

McHugo, G. J., Drake, R. E., Burton, H. L., et al (1995) A scale for assessing the stage of substance abuse treatment in persons with severe mental illness. Journal of Nervous and Mental Disease, 183, 762-767.

McLellan, A. T., Kushner, H., Metzger, D., et al (1992) The fifth edition of the Addiction Severity Index. Journal of Substance Abuse Treatment, 9, 199-213.

McLellan, A. T., Luborsky, L., Woody, G. E., et al (1980) An improved diagnostic evaluation instrument for substance abuse patients. The Addiction Severity Index. Journal of Nervous and Mental Disease, 168, 26-33.

Mechoulam, R., Parker, L. A. \& Gallily, R. (2002) Cannabidiol: an overview of some pharmacological aspects. Journal of Clinical Pharmacology, 42 (suppl. 11), 11-19.

Menezes, P. R., Johnson, S., Thornicroft, G., et al (1996) Drug and alcohol problems among individuals with severe mental illness in south London. British Journal of Psychiatry, 168, 612-619.

Miles, H., Johnson, S., Amponsah-Afuwape, S., et al (2003) Characteristics of subgroups of individuals with psychotic illness and a comorbid substance use disorder. Psychiatric Services, 54, 554-561.

Miller, W. R. \& Tonigan, J. S. (1996) Assessing drinkers' motivation for change: the Stages of Change Readiness and Treatment Eagerness Scale (SOCRATES). Psychology of Addictive Behaviors, 10, 81-89.

Moore, T. H. M., Zammit, S., Lingford-Hughes, A., et al (2007) Cannabis use and risk of psychotic or affective mental health outcomes: a systematic review. Lancet, 370, 319-328.

Mueser, K. T., Drake, R. E. \& Wallach, M. A. (1998) Dual diagnosis: a review of etiological theories. Addictive Behaviors, 23, 717-734.

Mueser, K. T., Yarnold, P. R., Rosenberg, S. D., et al (2000) Substance use disorder in hospitalized severely mentally ill psychiatric patients: prevalence, correlates and subgroups. Schizophrenia Bulletin, 26, 179-192.

Owen, R. R., Fischer, E. P., Booth, B. M., et al (1996) Medication non-compliance and substance abuse among patients with schizophrenia. Psychiatric Services, 47, 853-858.

Pencer, A., Addington, J. \& Addington, D. (2005) Outcome of a first episode of psychosis in adolescence: a 2-year follow-up. Psychiatry Research, 133, 35-43.

Potter, D. J., Clark, P. \& Brown, M. B. (2008) Potency of delta 9-THC and other cannabinoids in cannabis in England in 2005: implications for psychoactivity and pharmacology. Journal of Forensic Sciences, 53, 90-94.

Rey, J. M. \& Tennant, C. C. (2002) Cannabis and mental health. $B M], 325,1183-1184$.

Schofield, D., Tennant, C., Nash, L., et al (2006) Reasons for cannabis use in psychosis. Australian and New Zealand Journal of Psychiatry, 40, 570-574.

Sobell, L. C. \& Sobell, M. B. (1992) Timeline follow-back: a technique for assessing self-reported alcohol consumption. In Measuring Alcohol Consumption: Psychosocial and Biological Methods (eds R. Z. Litten \& I. Allen), pp. 41-72. Humana Press.

Sobell, L. C., Brown, J., Leo, G. I., et al (1996) The reliability of the Alcohol Timeline Followback when administered by telephone and by computer. Drug and Alcohol Dependence, 42, 49-54.

Sorbara, F., Liraud, F., Assens, F., et al (2003) Substance use and the course of early psychosis: a 2-year follow-up of firstadmitted subjects. European Psychiatry, 18, 133-136.

Spencer, C., Castle, D., Michie, P. T. (2002) Motivations that maintain substance use among individuals with psychotic disorders. Schizophrenia Bulletin, 28, 233-247.

United Nations Office on Drugs and Crime (2006) World Drug Report. UNODC (http://www.unodc.org/unodc/world_ drug_report.html).

van Os, J., Bak, M., Hanssen, M., et al (2002) Cannabis use and psychosis: a longitudinal population-based study. American Journal of Epidemiology, 156, 319-327.

Weaver, T., Renton, A., Stimson, G., et al (1999) Severe mental illness and substance misuse: research is needed to underpin policy and services of patients with comorbidity. BMJ, 318, 137-138.

Williams, S., Hickman, M., Bottle, A., et al (2005) Hospital admissions for drug and alcohol use in people aged under 45. BMJ, 15, 115.

Young, S. E., Rhee, S. H., Stallings, M. C., et al (2006) Genetic and environmental vulnerabilities underlying adolescent substance use and problem use: general or specific? Behavior Genetics, 36, 603-615.

Zammit, S., Allebeck, P., Andreasson, S., et al (2002) Self reported cannabis use as a risk factor for schizophrenia in Swedish conscripts of 1969. Historical cohort study. BMJ, 325, 1199-1201.

Zuardi,A. W.,Crippa,J.A.,Hallak, J.E., et al (2006) Cannabidiol, a Cannabis sativa constituent, as an antipsychotic drug. Brazilian Journal of Medical and Biological Research, 39, 421-429.

\section{MCQs}

1 Cannabis use:

a does not cause dependence

$\mathrm{b}$ is equally common among males and females

c is more common in people with severe mental illness

d significantly increases the risk of development of a psychotic illness in a non-dose-dependent manner

e is not associated with early psychotic illness.

2 Cannabis use by people with severe mental illness has been associated with:

a decreased rate of suicide

$\mathrm{b}$ decreased risk of violence

c decreased risk of victimisation

d increased risk of weight gain

e homelessness.

3 Of the following, the most likely reason that people with severe mental illness use cannabis is that:

a it helps to treat their negative symptoms

$\mathrm{b}$ it reduces their social interaction

c it increases their dysphoria

$\mathrm{d}$ one of the cannabis compounds may be treating their psychotic symptoms

e they need to self-medicate.

4 In assessing a patients' cannabis use the following points are not important:

a which part of cannabis plant they use

b stressful events

c the severity of psychotic symptoms

$\mathrm{d}$ the effect of use in relationships

e whether the individual eats cheese while using. 


\section{As regards cannabis:}

a its use is more common in low-income countries, because it is cheap and available

b use can be controlled only through strict legislation

c people with high schizotypal personality scores are not likely to report unpleasant after-effects associated with cannabis use

d several models have been proposed to explain the complex relationship between cannabis use and severe mental illness

e use does not increase the risk of cancer.

\section{MCQ answers}

$\begin{array}{lllll}1 & 2 & 3 & 4 & 5\end{array}$

a F a F a F a F a F

b F b F bF b F b F

c $\mathrm{T}$ c F c F c F c F

d F d F d T d F d T

e $F$ e $\mathrm{T}$ e $\mathrm{F}$ e $\mathrm{T}$ e $\mathrm{F}$

